\title{
SCALABLE CASCADED SNAP-IN ACTUATORS FOR LARGE-STROKE DISPLACEMENTS
}

\author{
N. Golay ${ }^{\text {I) }}$, A. Masse $^{2)}$, Y. Pétremand ${ }^{1)}$,W. Noell ${ }^{1)}$, J.-F. Manceau ${ }^{2)}$, and N.F. de Rooij ${ }^{1)}$ \\ ${ }^{1}$ Ecole Polytechnique Fédérale de Lausanne (EPFL), Institute of Microengineering (IMT), Sensors, Actuators \\ and Microsystems Laboratory, Breguet 2, 2000 Neuchâtel, Switzerland \\ ${ }^{2}$ FEMTO-ST, University of Franche-Comté, Besançon, FRANCE
}

\begin{abstract}
This paper will focus on the design, and first measurements of a cascaded in-plane parallel plate snap-in actuator. The actuator is based on a rather simple microfabrication process and can achieve a total displacement of several tenths of microns. Compared to classical noncascaded transducer device based on parallel plates or comb-drive actuator, the actuation voltage is relatively low due to the snap-in phenomenon of electrostatic actuators. The electromechanical response of such a device is sequential. The fabricated 4-stage device shows a total stroke of $75 \mu \mathrm{m}$ at $60 \mathrm{~V}$. It is possible to easily increase the total stroke of the actuator by increasing the number of stages. Only one input electrode is required. Simulations with CoventorWare showed easy scalability of the concept for up to 19 stages with a total displacement of $350 \mu \mathrm{m}$.
\end{abstract}

\section{KEYWORDS}

Cascaded actuator, parallel plate actuator, delayed mask process, electrostatic force, snap-in actuation, large stroke

\section{INTRODUCTION}

Standard parallel plate actuators are limited in maximum displacement due to the small gap between the electrodes. To be able to have larger displacements, the gap has to be larger and the potential to activate the device has to be increased, although that requires a higher electrostatic voltage to obtain the same force.

The presented device uses a cascaded configuration of snap-in actuators. The cascaded structure combines the advantage of increasing the total stroke of the structure with a relatively low initial gap and relatively low actuation voltage. The system is a compromise between a classical capacitor plate actuator and a zipper actuator $[1,2]$. Since the system's response is sequential, it can be used as an analog to digital converter (AD).

The fabrication is based on a single mask process for the front-side and a delayed-mask process for the backside. The device is micro-fabricated on SOI wafers by double-side etching with Deep Reactive Ion Etching (DRIE). The release and singulation of the device is made by vapor phase hydrofluoric acid etching (HF VPE) [3].
No additional layers or filling processes are required for electrical and mechanical connections, between the different stages of the cascaded actuator.

\section{DEVICE STRUCTURE AND PRINCIPLE}

Figure 1 shows the electromechanical concept of a 2stage device. The first electrode is connected to ground and the second one is connected to the voltage source. The third plate electrode is electrically linked to the first one by the U-shape spring system, and mechanically coupled to the second one by a thinned down block located underneath and fabricated from the handle layer of the SOI wafer. The buried oxide of the SOI wafer acts as an electrical insulator between the electrodes \#2 and \#3. These first two electrodes compose the first stage of the cascaded structure. The connection between each stage is identical to the ones just described. To drive the whole structure, a hook system is implemented at the end of each U-shape spring, which takes the next stages along and permits to have a constant gap between each stage. Figure 2 shows the hook system.

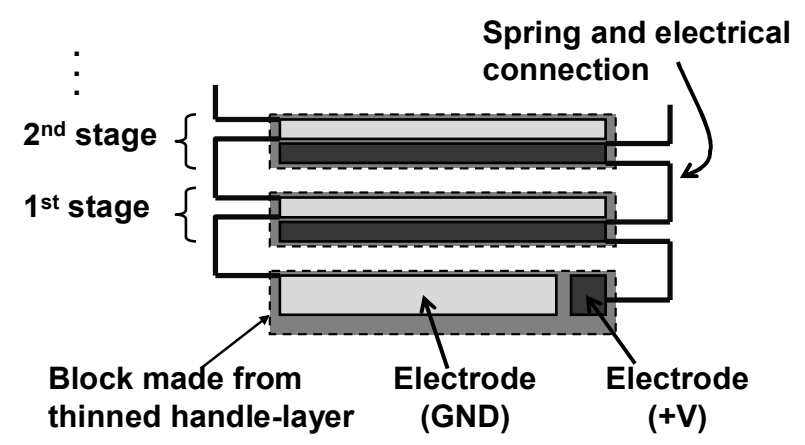

Figure 1: Concept of a 2-stage scalable design. Each stage contains two electrodes that are mechanically connected by a backside block. The U-springs make the electrical connection between the stages, either GND (left side) or +V (right side).

The solution permits to snap-in all stages when the first one is activated. To be able to sequentially activate each stage, the spring constant is increased from stage to stage by reducing the length of the bent beam of the Ushape spring. Under each springs (particularly under the $+\mathrm{V}$ springs) the handle layer is completely removed to avoid short-cut between the spring and the handle layer due to the difference of electrical potential. 


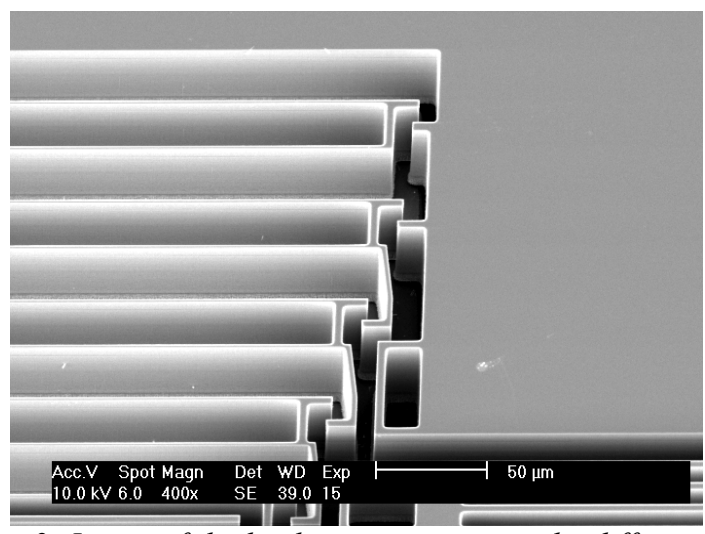

Figure 2: Image of the hook system retaining the different stage at the end of each springs

In order to have lateral stability and maintain the total mobile structure, two soft U-shape springs are added on the last stage of the cascaded actuator. These springs are electrically insulated for the rest of the design by a trench around the fixed anchor.

To avoid short-cut between the electrodes, a stopper is added to each corner of the electrode. To have a good stability the electrode has a shape of an L. Each electrode has only contact with another electrode with the same electrical potential. The contact area is minimized to avoid the risk of stitching between electrodes when the actuator is closed.

Six different structures were designed and simulated using CoventorWare finite elements analysis. These structures are composed of different number of stages and electrodes thickness. Figure 3 shows SEM pictures of a part of the chip. It is possible to observed the different design (electrode thickness and number of stages)

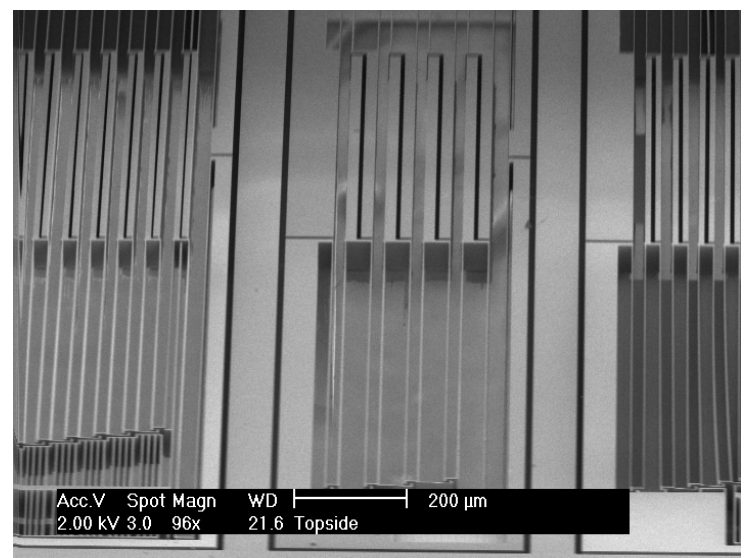

Figure 3: Image of a part of the actuator. Several parallel plate actuators - with up to 19 stages - were micro-fabricated and electrically tested.
The simulation shows the required potential to activate all the 3 -stages of the actuator. The first stage is activated with a potential of $20 \mathrm{~V}$. The second stage snaps-in at $45 \mathrm{~V}$, and finally the last stage snaps-in at $60 \mathrm{~V}$. The displacement of each stage is $20 \mu \mathrm{m}$. The total stroke obtained with the simulation is $60 \mu \mathrm{m}$. Figure 4 presents the results given by the FEM analysis.

a)

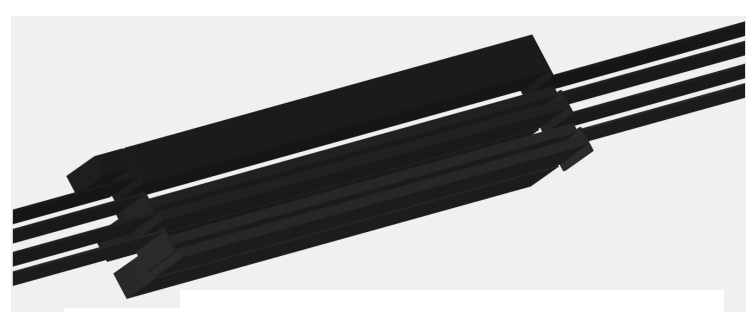

b)

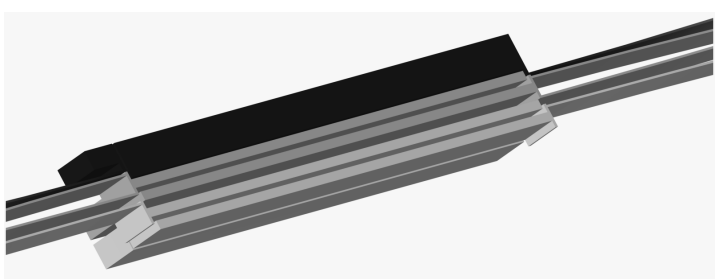

c)

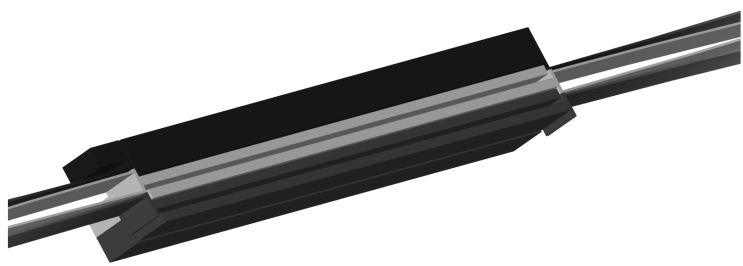

Figure 4: FEM simulation of a 3 stages snap-in actuator. a) The first stage is activated with a potential of $20 \mathrm{~V}$, b) the second stage snaps-in at $45 \mathrm{~V}, \mathrm{c}$ ) the third stage snaps-in at a voltage of $60 \mathrm{~V}$. 


\section{FABRICATION PROCESS}

To realize this actuator, the micro-fabrication process used is based on double-side etching process. An SOI wafer with a device layer thickness of $50 \mu \mathrm{m}$ is used. The electrodes and the U-shape springs are built in the device layer using photolithography and deep reactive ion etching process (DRIE). Figure 5 shows the main step of the micro-fabrication process.

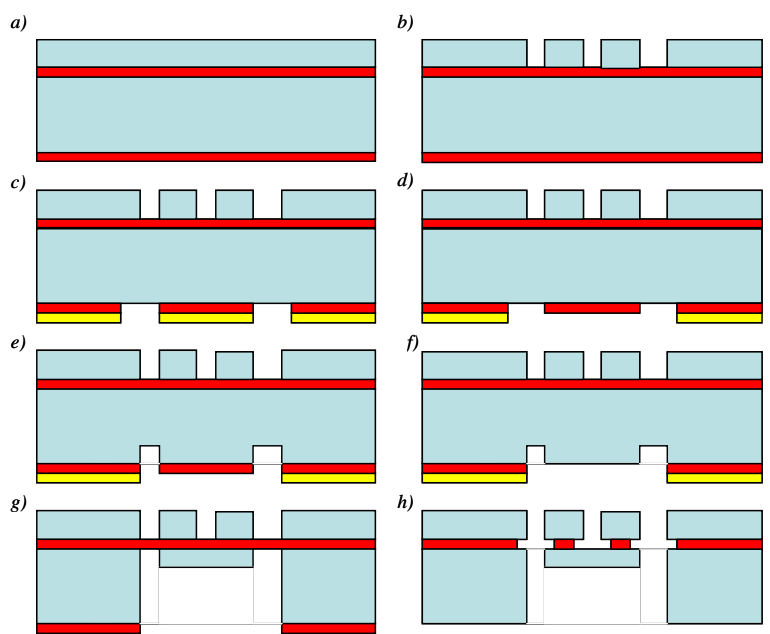

Figure 5 Process flow used for the fabrication of the device. a) $1^{\text {st }}$ photolithogryphyof the device layer, b) DRIE of the device layer, c) structuring of the $\mathrm{SiO}_{2}$ of the handle layer, d) photolithography of the delayed mask process, e) $1^{\text {st }}$ step of etching of the handle layer, f) etch in $\left.\mathrm{SiO}_{2}, g\right)$ thinning down the handle layer, $h$ ) release the mobile structure with $H F$ vapor.

To build the handle layer, a delay mask etching process is used. The first step of the fabrication of the handle layer is a photolithography on a thin layer of photoresist to structure the silicon dioxide layer of the back-side of the handle layer. To etch the $\mathrm{SiO}_{2}$ layer, a wet process composed of BHF acid is used.

After the pattering of the silicon dioxide, the photoresist is removed by oxygen plasma. The second photolithography is carried out on thick photoresist layer. The first step of the handle layer is etched. After obtaining the required step height, the resist is removed by oxygen plasma. The second step of etching is using the silicon dioxide layer as the protective etch mask to thin down the handle layer until the right thickness. The major point in the delay mask process of the handle layer was to conserve a relatively small gap between each stage in the handle layer. Figure 6 shows a SEM picture of the back-side of the actuator. It is possible to observe the two etch heights of the handle layer.

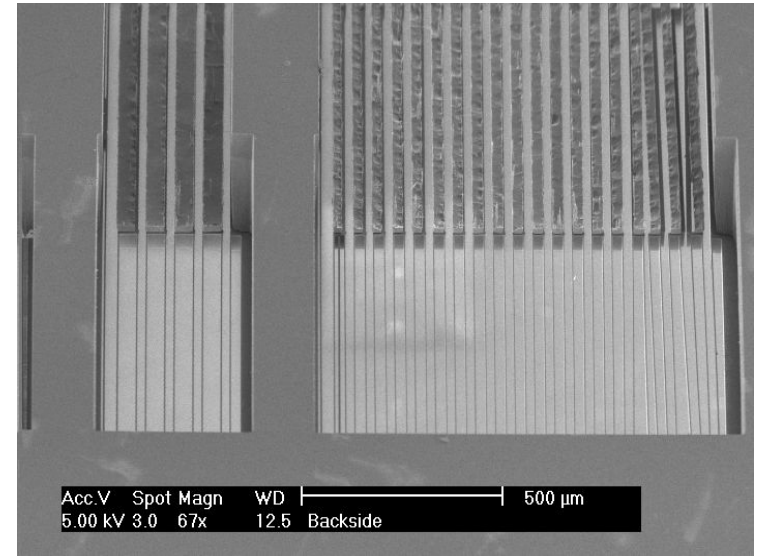

Figure 6: Image of the back side after the delay mask process. The two electrodes are fixe by a thinned down handle layer

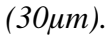

The release step by vapor phase hydrofluoric acid is critical part of the process. On the one hand the structure has to be mobile but on the other hand, the electrodes have to be mechanically fixed to the silicon dioxide. During the release phase, the chip is directly singulated from the wafer frame. With this technique, the problem of having fragile parts and dust between the electrodes occurring during classical dicing process is minimized.

\section{EXPERIMENTAL RESULT}

Several devices were fabricated from a $100 \mathrm{~mm}$ SOI wafer using DRIE technology. Chips were characterized electromechanically under a prober station. The applied voltage comes from a voltage source with a current limitation of $100 \mu \mathrm{A}$. First tests were made on a single stage chip. Simulation made on single stage device show an actuation voltage of $16 \mathrm{~V}$ to have snap-in occurs.

The goal of this experiment was to verify the possibility to realize a functional stage of electrodes. The second experiment was the proof-of-concept of a multi stage device. To test this structure, the applied voltage is progressively increased until the first stage snaps-in. The hook system retaining the different stages together, show the functionality and efficiency of the concept. By increasing the voltage progressively, the different stages of the actuator are activated sequentially. The snap-in of each stage appeared at a different voltage as predicated by the simulations. 
To measure the displacement of each stage, chips were wire-bonded and placed under a white light interferometer (Wyko). Figure 8 presents the results obtained from a 4stages configuration actuator under Wyko measurement. The first stage is activated with a voltage of $20 \mathrm{~V}$. The stroke of the structure is $20 \mu \mathrm{m}$. By applying a potential of $30 \mathrm{~V}$ the second stage snaps to the first one and the total movement of the mobile part is $38 \mu \mathrm{m}$. A displacement of $55 \mu \mathrm{m}$ is observed when the third stage is actuated at $38 \mathrm{~V}$. The total stroke measured on this structure is $75 \mu \mathrm{m}$ at $60 \mathrm{~V}$. Compare to simulation, the result obtain are in the same order of magnitude. The main difference between simulated structure and real measurements device comes from the micro-fabrication process. A small overetch of each beam, changes the actuation voltage due to the variation of the spring constant.

It is possible to observe the displacement of each stage measured individually versus the applied voltage. The sequential displacement of each stage is clearly visible and shows a step-wise response to an analog and continuously increasing input voltage ramp. This result shows the capability of the system to be used as an $\mathrm{AD}$ converter.

Figure 7 shows the image taken under the prober during the testing phase.
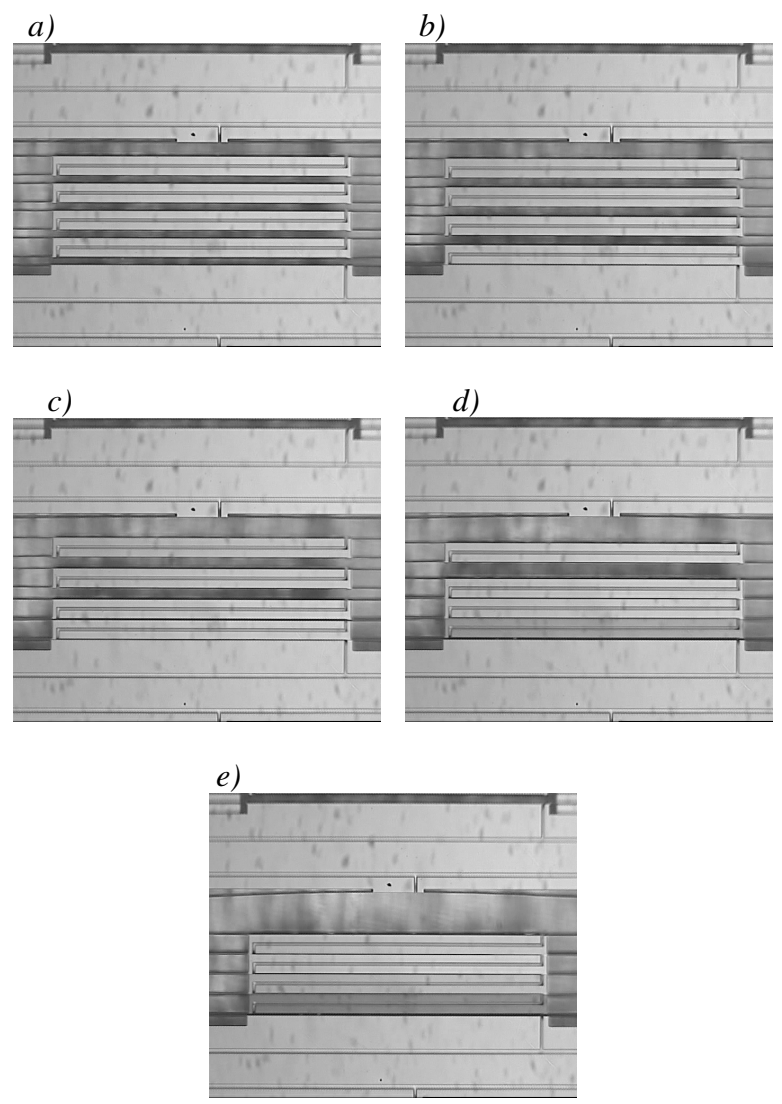

Figure 7: Actuation sequence of the 4 different stages of the snap-in actuator.

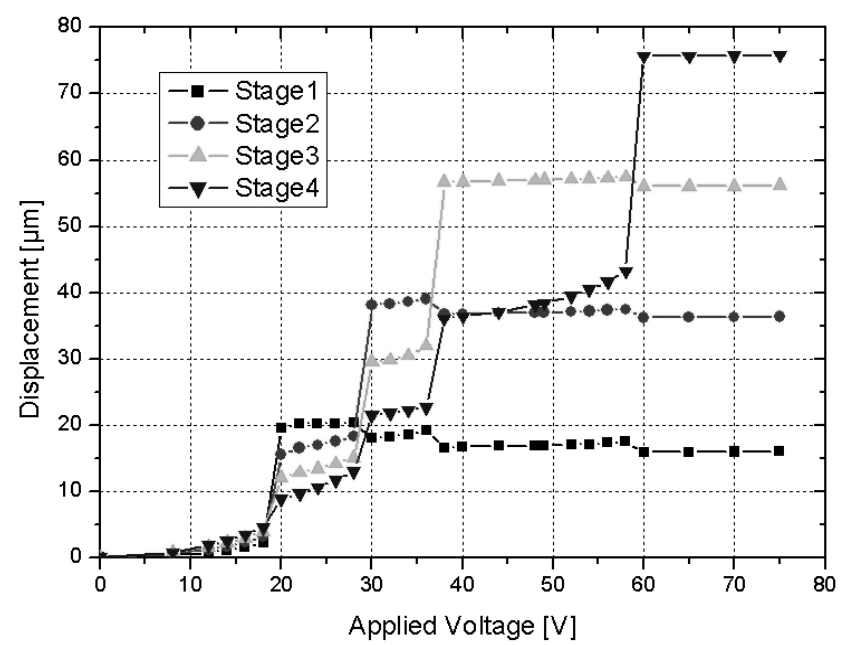

Figure 8: Displacement of each stage versus the applied voltage. The maximum stroke is about $75 \mu \mathrm{m}$ at 60 . All stages are connected through a single electrode (cf. Fig. 1).

\section{CONCLUSION}

The concept and feasibility of a cascaded snap-in actuator is demonstrated. Results show the capability of the system to be scalable for extremely large in-plane strokes at a relatively small footprint and for reasonably low applied voltages. The device shows the potential for a MEMS-based analog to digital converter and has the possibility to be used as a tunable grating for optical setups.

\section{ACKNOWLEDGMENTS}

We gratefully acknowledge all the COMLAB staff for the help and knowhow to realize this project.

\section{REFERENCES}

[1] Chiou J.C., Kuo C.F., Lin Y.J., Chang C.W. and Hou K.C. "Development of novel cascade structure for improving stroke of electrostatic comb-drive actuator", IEEE, MEMS 2008.

[2] Li J., Brenner M. P., Christen T., Kotilainen M. S., Lang J. H., Slocum A. H. "Deep-Reactive Ion-Etched Compliant Starting Zone Electrostatic Zipping Actuator", Journal Of Microelectrical Systems, Vol. 14, $\mathrm{N}^{\circ} 6$, December 2005.

[3] T. Overstolz, P. A. Clerc, W. Noell, M. Zickar, and N. F. de Rooij, "A Clean Wafer-Scale Chip-Release Process without Dicing Based on Vapor Phase Etching, Digest of the $17^{\text {th }}$ IEEE Int. Conf. On Micro Electro Mechanical Systems, pp. 717-720, 2004. 